\title{
Análisis florístico en tepetates del estado de Tlaxcala
}

\section{Floristic analysis in tepetates of Tlaxcala State}

\author{
José Luis Martínez-y-Pérez ${ }^{1 *}$, Gonzalo Castillo-Campos², Ma. Guadalupe Santiago-Martínez y Laura V. \\ Hernández-Cuevas ${ }^{3}$ \\ ${ }^{I}$ Laboratorio de Sistemática, Centro de Investigación en Ciencias Biológicas, Universidad Autónoma de Tlaxcala. Km. 10.5 Autopista Tlaxcala-Tex- \\ melucan, Ixtacuixtla 90022 Tlaxcala, México. \\ ${ }^{2}$ Departamento de Biodiversidad y Sistemática, Instituto de Ecología, A.C. Km. 2.5 Carretera antigua a Coatepec, Xalapa 91070 Veracruz, México \\ ${ }^{3}$ Laboratorio de Micorrizas, Centro de Investigación en Ciencias Biológicas, Universidad Autónoma de Tlaxcala. Km. 10.5 Autopista Tlaxcala-Tex- \\ melucan, Ixtacuixtla 90022 Tlaxcala, México \\ *Correspondencia: jlmarpe@hotmail.com
}

\begin{abstract}
Resumen. En México no se conocen trabajos sobre florística de los tepetates, ya que se consideran suelos improductivos y poco interesantes para su estudio. Se recolectó material botánico durante 18 meses en 2 localidades del estado de Tlaxcala con presencia de tepetates y en 40 unidades experimentales con barreras para retención de sedimentos y 40 sin barreras, que también fueron utilizadas para introducir arbustos nativos micorrizados. Las plantas se identificaron taxonómicamente y se depositaron en el herbario TLXM. Se registran 44 familias, 106 géneros y 140 especies que representan más del 10\% de lo conocido para el estado. Las 2 localidades comparten 51 especies y existen muchas exclusivas de cada sitio. El análisis de varianza muestra diferencias significativas $(\mathrm{p}<0.05)$ en el número de especies entre localidades y entre unidades experimentales con y sin barreras. El análisis de semejanza con el método UPGMA y el coeficiente de Baroni-Urbani y Buser permiten proponer 4 grupos de las unidades experimentales con $43 \%$ de semejanza; 2 corresponden a unidades con pocas especies; y cada uno de los otros 2 agrupan, respectivamente, la mayoría de las unidades de Atlangatepec y las de Altzayanca, lo que puede deberse a la presencia de las familias Asteraceae y Poaceae, consideradas de amplia distribución y afines a ambientes deteriorados. Se registra Coryphanta bussleri (Mundt) Scheinvar por primera vez para Tlaxcala, lo cual representa una nueva población para México.
\end{abstract}

Palabras clave: plantas, semejanza florística, suelos degradados.

\begin{abstract}
We present one floristic analysis of tepetates (indurated volcanic soils) in Tlaxcala State because information of these kind environments is poor in Mexico. We recollected botanic samples of vascular plants during 18 months in 2 localities of Tlaxcala State with volcanic soils. We used 40 experimentation parcels with borders for sediments accumulation and 40 without them used for introduced natives shrub micorrized. Vegetational samples were identified taxonomically and deposited in TLXM herbaria. We listed 44 families, 106 genera and 140 species that represent more of $10 \%$ known flora for the state. There are 51 species common for the 2 localities and the majority of them are exclusively at their own localities. There are significatives differences $(p<0.05)$ between localities and between experimentation parcels with borders or without. The analysis shows 4 groups with $43 \%$ of similarity, the first and second represent the parcels with few species, the third and fourth represent parcels of Atlangatepec and Altzayanca respectively, this situation could be influenced by the presence of Asteraceae and Poaceae, families which are known of wide distribution and with affinities to open enviroments. Fouthermore, we reported a new population for Coryphanta busleri (Mundt) Scheinvar for Mexico.
\end{abstract}

Key words: degradated soils, floristic similarity, plants.

\section{Introducción}

A los suelos volcánicos endurecidos en México se les denomina tepetates, se conocen desde la época prehispánica y su nombre deriva del vocablo náhuatl tepetlatl, que alude

Recibido: 08 enero 2010; aceptado: 04 octubre 2010 a una capa endurecida del suelo (Gama-Castro et al., 2007). El estudio de los tepetates se ha abordado desde diferentes perspectivas y en cuanto a su origen, se mencionan 3 formas principales: 1) geológica, mediante el intemperismo del material volcánico; 2) diagenético-pedológica, por la hidroconsolidación o silificación de la ceniza volcánica, y 3) degradación física del suelo, la cual puede ser natural por efecto de las lluvias, compactación, consolidación, cementación, o por las actividades antropógenas (Werner, 2005; 
Gama-Castro et al., 2007; Haulon et al., 2007).

Se estima que en México, los tepetates ocupan una extensión aproximada de 22235474 ha (INEGI, 2003), ubicándose principalmente en la parte central y el norte del país, aunque también se presentan en la península de Baja California. Dada su extensión, se han propuesto varias acciones para reincorporarlos a las actividades agrícolas, mediante roturación (Prat et al., 2003; Werner, 2005) e incorporación de materia orgánica (Betancourt et al., 1999; Álvarez-Solís et al., 2000; Baéz-Pérez et al., 2002; GamaCastro et al., 2007; Haulon et al., 2007; García-Cruz et al., 2008; Velázquez-Rodríguez et al., 2008; Pajares-Moreno et al., 2010).

El área ocupada por tepetates en Tlaxcala representa el $54 \%$ de la superficie total del estado y se considera improductiva. Estos tepetates se han originado por degradación física ocasionada por el desarrollo de monocultivos; corresponden al tipo fragipan (Navarro-Garza et al., 2004) y presentan cárcavas que van de someras a profundas (Guerra, 2005).

Se han realizado investigaciónes con la finalidad de incorporarlos a la producción agrícola (Werner, 2005; Haulon et al., 2007) y también para estimar la pérdida de la capa orgánica superficial de los suelos por arrastre de las corrientes de agua y el consecuente afloramiento de los tepetates, erosionando aproximadamente $354000 \mathrm{t}$ año ${ }^{-1}$ de suelo (Betancourt et al., 2000; Návar y Sinnot, 2000; Guerra, 2005).

Asimismo, se han estado realizando estudios que buscan la recuperación del suelo de estos tepetates mediante la utilización de plantas adaptadas a condiciones secas y asociadas con microorganismos benéficos, tales como los hongos micorrizógenos (Campos-Mota et al., 2004; GonzálezChávez et al., 2004; Loredo-Osti et al., 2004; García-Cruz et al., 2007, 2008; Pérez, 2009; Rodríguez, 2009). Sin embargo, no existen trabajos que mencionen las especies vegetales nativas capaces de desarrollarse en este tipo de suelos y que puedan utilizarse para rehabilitar los tepetates. Por tal motivo, el objetivo de este estudio fue conocer la flora nativa presente en 2 localidades con tepetates y analizar las diferencias y las semejanzas en número de especies de ambas localidades y además proveer información sobre las especies de plantas nativas que están asociadas con las plantas micorrizadas introducidas.

\section{Materiales y métodos}

El estudio se realizó en 2 localidades ubicadas en los municipios de Altzayanca y Atlangatepec, en el estado de

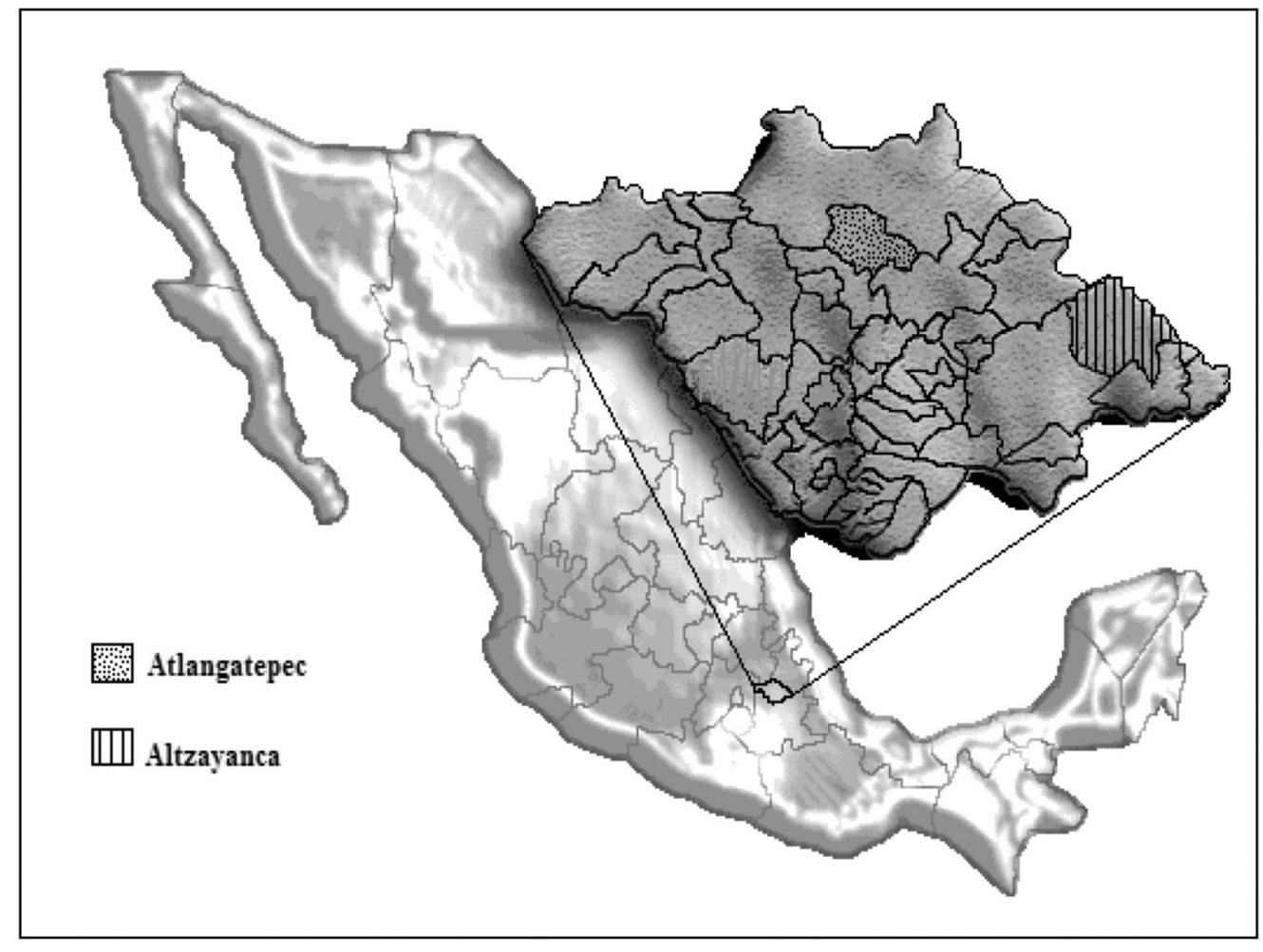

Figura 1. Ubicación de las localidades estudiadas. 
Tlaxcala (Fig.1). El área aproximada para cada localidad con tepetate expuesto es de 1 ha para Altzayanca (AL), rodeada de bosque de pino (Pinus pseudostrobus Lindl.) y a $2600 \mathrm{~m} \mathrm{snm}$, y de 3 ha para Atlangatepec (AT), rodeada por bosque de Juniperus deppeana Steud. (Rzedowski de y Rzedowski, 2005) y a $2300 \mathrm{~m}$ snm. En cada localidad se delimitaron 40 unidades de experimentación de 4 x 4 m; una con barreras hacia la parte baja de la pendiente para la retención del suelo superficial que se pierde por efecto del escurrimiento del agua de lluvia (R); la otra sin barreras (NR). Ambas contiguas (Fig. 2). En cada unidad experimental fueron sembradas 20 plántulas de las siguientes especies: Pinus cembroides Zucc. y Comarostaphylis discolor (Hook.) Diggs, micorrizadas con Suillus glandulosipes Thiers et A. H. Sm., S. tomentosus (Kauffman) Singer, Snell et Dick e Inocybe dulcamara (Alb. et Schw.) Kumm, además de plántulas de Amelanchier denticulata (Kunth) K. Koch y Eysenhardtia polystachya (Ortega) Sarg. micorrizadas con Glomus claroideum Schenck et Smith, Acaulospora laevis Gerd. et Trappe y A. morrowae Spain et Schenck (SantiagoMartínez, 2010).
La recolección de todas las plantas vasculares se realizó de manera intensiva cada 30 días, de enero de 2007 a julio de 2008, tanto en el área total de tepetates como en cada una de las unidades de experimentación de ambas localidades. El material recolectado fue herborizado para su identificación en el Laboratorio de Sistemática del Centro de Investigación en Ciencias Biológicas de la Universidad Autónoma de Tlaxcala, con las claves de identificación de la Flora Fanerogámica del Valle de México (Rzedowski de y Rzedowski, 2005), Flora Mesoamericana (Davidse et al., 1994) y las claves monográficas de algunos grupos de plantas. La identificación de los helechos se realizó con las claves de Pteridophyta de la Flora Novogaliciana (McVaugh, 1992).

El material recolectado e identificado ha sido depositado en la colección del herbario universitario TLXM para su respaldo. La información obtenida a través del inventario florístico se organizó en una lista, cuadros y gráficas para ambas localidades, por taxa, tanto del área total de tepetates como para las unidades experimentales. Con la información del número de especies para cada unidad experimental se realizó un análisis de varianza, para evaluar diferencias

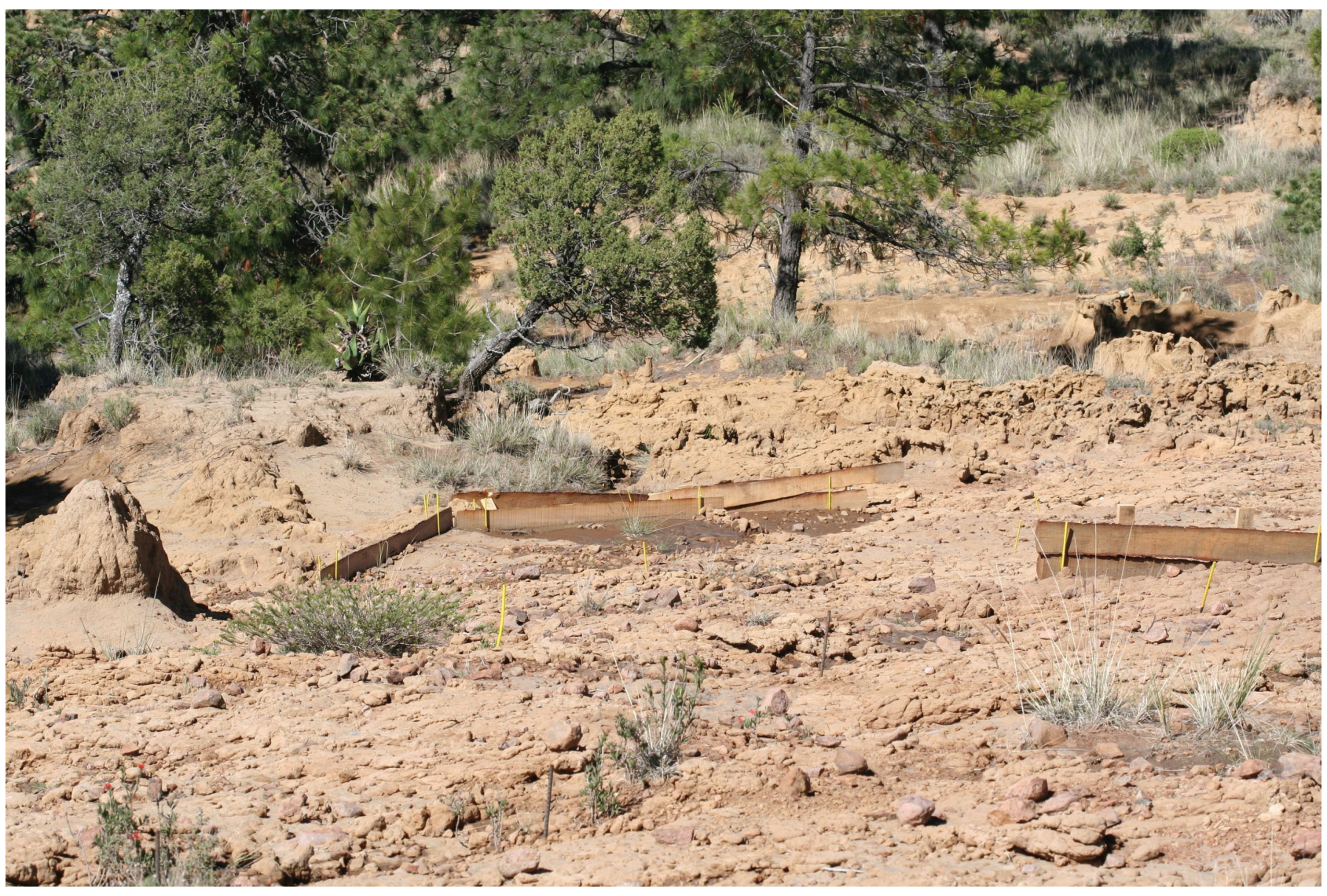

Figura 2. Unidades experimentales con barreras (al fondo) y sin barreras. 
entre localidades y unidades de experimentación, con y sin barreras, mediante el programa Statistica (StatSoft, 1998). Utilizando la presencia-ausencia de las especies en cada unidad experimental se hizo un análisis de semejanza, con el método UPGMA y el coeficiente de Baroni-Urbani y Buser (1976) en el programa MVSP ver. 3.11 (Kovach Computing Services, 2000).

\section{Resultados}

Se registraron para ambas localidades 140 especies de 106 géneros y 44 familias de plantas vasculares (Apéndice 1), lo que representa aproximadamente el $34.92 \%$ de las familias, el $20.92 \%$ de géneros y el $11.63 \%$ de las especies conocidas para el estado de Tlaxcala (Acosta et al., 1991; Castillejos et al., 2007); y el $63.76 \%$ de las familias, el $49.76 \%$ de los géneros y el $35.14 \%$ de las especies mencionadas para el Parque Nacional La Malinche (López et al., 2005), una de las áreas de vegetación más importantes del estado, que ha sido explorada extensivamente.

Se registraron 35 especies que no se encuentran representadas en las unidades experimentales, existen 51 especies exclusivas para la localidad de Atlangatepec y 30 en Altzayanca. Las especies compartidas entre ambas localidades son 51. Las especies exclusivas en las unidades experimentales de Atlangatepec son 38 y 31 para Altzayanca y comparten 36 especies.

Con base en el índice de semejanza, las parcelas contiguas con barreras o sin ellas no necesariamente presentan los valores más altos (Fig. 3); sin embargo, se puede observar que a un nivel del $43 \%$ de semejanza (línea de corte) se resuelven 4 grupos, el primero y el segundo representan unidades experimentales con sólo 1 especie registrada para cada una, el tercer grupo relaciona unidades experimentales de la localidad de Altzayanca y el cuarto grupo relaciona unidades experimentales de la localidad de Atlangatepec, aunque se adicionan a este grupo 2 unidades experimentales de Altzayanca (ALNR3 y ALR2, con y sin barreras, no contiguas). Una unidad experimental en la localidad de Altzayanca (ALR3, sin barrera) no mostró ninguna especie durante la realización del presente trabajo y no fue incluida en el análisis de semejanza.

Las familias más diversas en ambas localidades son Poaceae con más de 26 especies y Asteraceae con más de 20 especies (Fig. 4). El mayor número de especies estacionales para ambas localidades se desarrolla en los meses de agosto y septiembre lo cual coincide con la mayor cantidad de precipitación (época de lluvias o también llamada localmente como "de la verdura"). En general, las unidades de experimentación sin barreras presentaron menor número de especies comparadas con las que si la tenían (Cuadro 1), obteniéndose diferencias significativas entre el número de especies por localidad como entre las unidades experimentales con y sin barre$\operatorname{ras}(F=60.32765, \mathrm{p}<0.00001 ;$ Fig. 5).

\section{Discusión}

El inventario obtenido para suelos degradados resulta interesante desde el punto de vista florístico debido a que en las áreas estudiadas está representado más del 10\% de las especies listadas para el estado de Tlaxcala (Castillejos et al., 2007) y el 35\% de las conocidas para el Parque Nacional La Malinche (López et al., 2005), lo cual refuerza los comentarios vertidos por dichos autores de que las zonas secas están poco exploradas; por lo tanto, la riqueza de especies registrada de tales áreas podría incrementarse conforme se tengan más estudios; por ejemplo, en este trabajo se registraron especies que no se habían mencionado para el estado de Tlaxcala (Acosta et al., 1991; Sánchez, 1993; Castillejos et al., 2007).

La presencia de especies anuales en los tepetates (Apéndice 1) adaptadas a sobrevivir en condiciones ambientales extremas, con sus semillas capaces de estar en estado de latencia hasta por 20 años (Tsuyuzaki y Goto, 2001), como puede estar sucediendo con los representantes de las familias Asteraceae y Poaceae, las cuales varios autores las consideran como grupos de especies pioneras (Cain et al., 2000), representan una buena oportunidad para ser utilizadas en programas de recuperación de estas áreas, o bien, son potencialmente modelos de estudios para información molecular sobre resistencia a condiciones extremas.

La presencia de 35 especies no encontradas en las unidades de experimentación, pero sí en los suelos con tepetates, se debe a que muchas de ellas se recolectaron cerca de los bordes de las áreas con vegetación que rodean los tepetates, y posiblemente sus estructuras reproductivas no llegan a los suelos erosionados o no son capaces de desarrollarse en ambientes extremos. Es notable el alto porcentaje (casi 50\%) de especies catalogadas como exclusivas en cada localidad estudiada y que puede estar dado por influencia del tipo de vegetación, pendiente del suelo, humedad prevaleciente y características ambientales y microbiota diferentes entre ellas (Pérez, 2009).

Con base en lo precedente, los valores obtenidos en el análisis de semejanza demuestran que hay muy pocas especies compartidas entre localidades; tal es el caso de las unidades experimentales ALR2 y ALNR3 de Altzayanca (con y sin barreras, unidades no contiguas) que forman un pequeño subgrupo con una unidad de Atlangatepec (ATR13, sin barrera), dentro del grupo 4 de Atlangatepec 


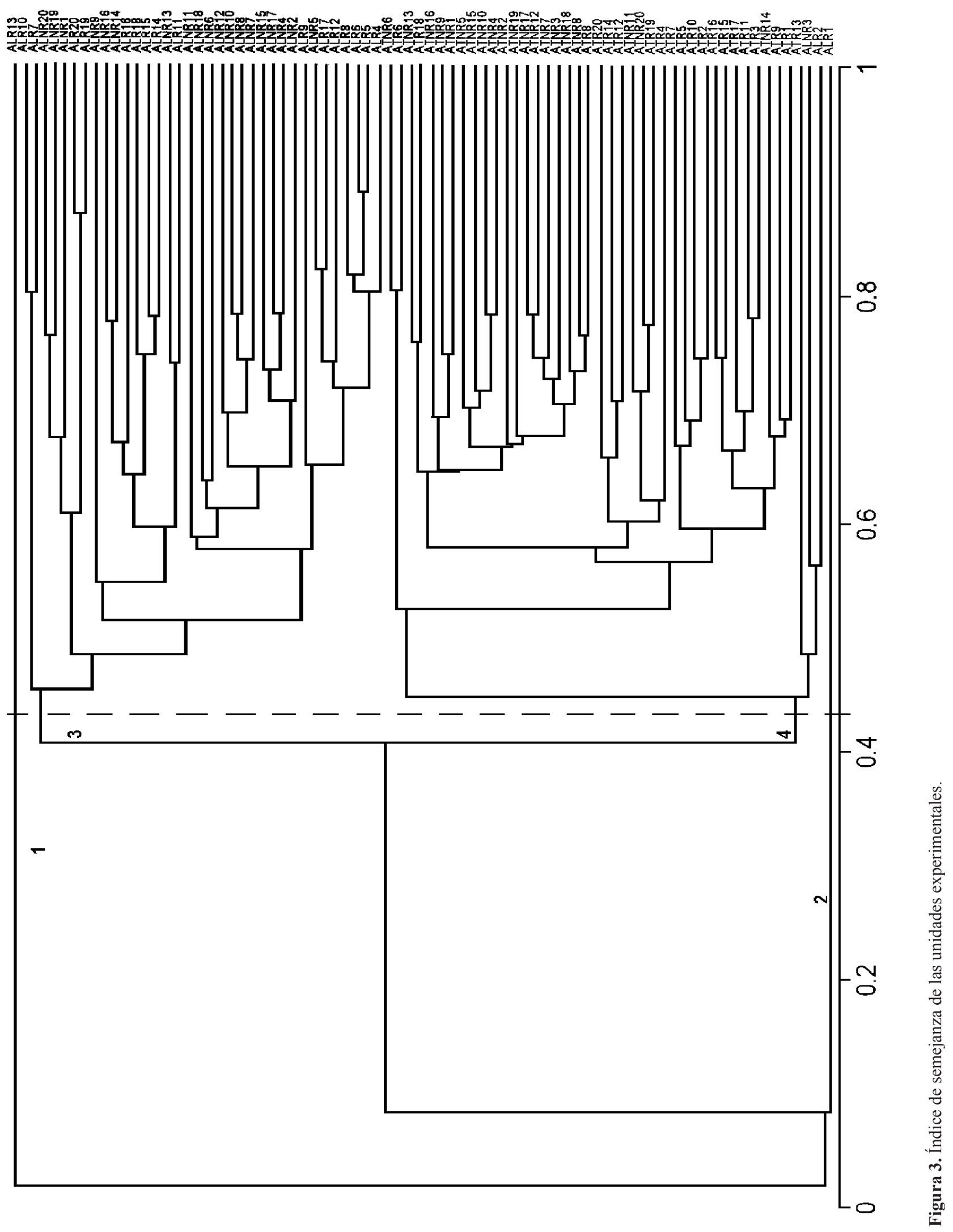


Cuadro 1. Número total de especies observadas en cada unidad experimental

\begin{tabular}{|c|c|c|c|c|}
\hline \multirow{2}{*}{$\begin{array}{l}\text { Núm. de unidad } \\
\text { experimental }\end{array}$} & \multicolumn{2}{|c|}{ Altzayanca } & \multicolumn{2}{|c|}{ Atlangatepec } \\
\hline & Sin barrera & Con barrera & Sin barrera & Con barrera \\
\hline 1 & 1 & 13 & 16 & 29 \\
\hline 2 & 3 & 14 & 9 & 19 \\
\hline 3 & 0 & 13 & 12 & 24 \\
\hline 4 & 5 & 23 & 22 & 21 \\
\hline 5 & 2 & 11 & 12 & 27 \\
\hline 6 & 4 & 12 & 19 & 23 \\
\hline 7 & 4 & 14 & 10 & 26 \\
\hline 8 & 6 & 18 & 22 & 28 \\
\hline 9 & 7 & 19 & 18 & 25 \\
\hline 10 & 4 & 18 & 10 & 20 \\
\hline 11 & 7 & 18 & 11 & 22 \\
\hline 12 & 4 & 13 & 17 & 22 \\
\hline 13 & 1 & 6 & 9 & 24 \\
\hline 14 & 14 & 19 & 13 & 21 \\
\hline 15 & 9 & 19 & 10 & 23 \\
\hline 16 & 13 & 20 & 9 & 24 \\
\hline 17 & 10 & 18 & 16 & 24 \\
\hline 18 & 6 & 9 & 24 & 29 \\
\hline 19 & 8 & 18 & 9 & 21 \\
\hline 20 & 6 & 14 & 15 & 17 \\
\hline
\end{tabular}

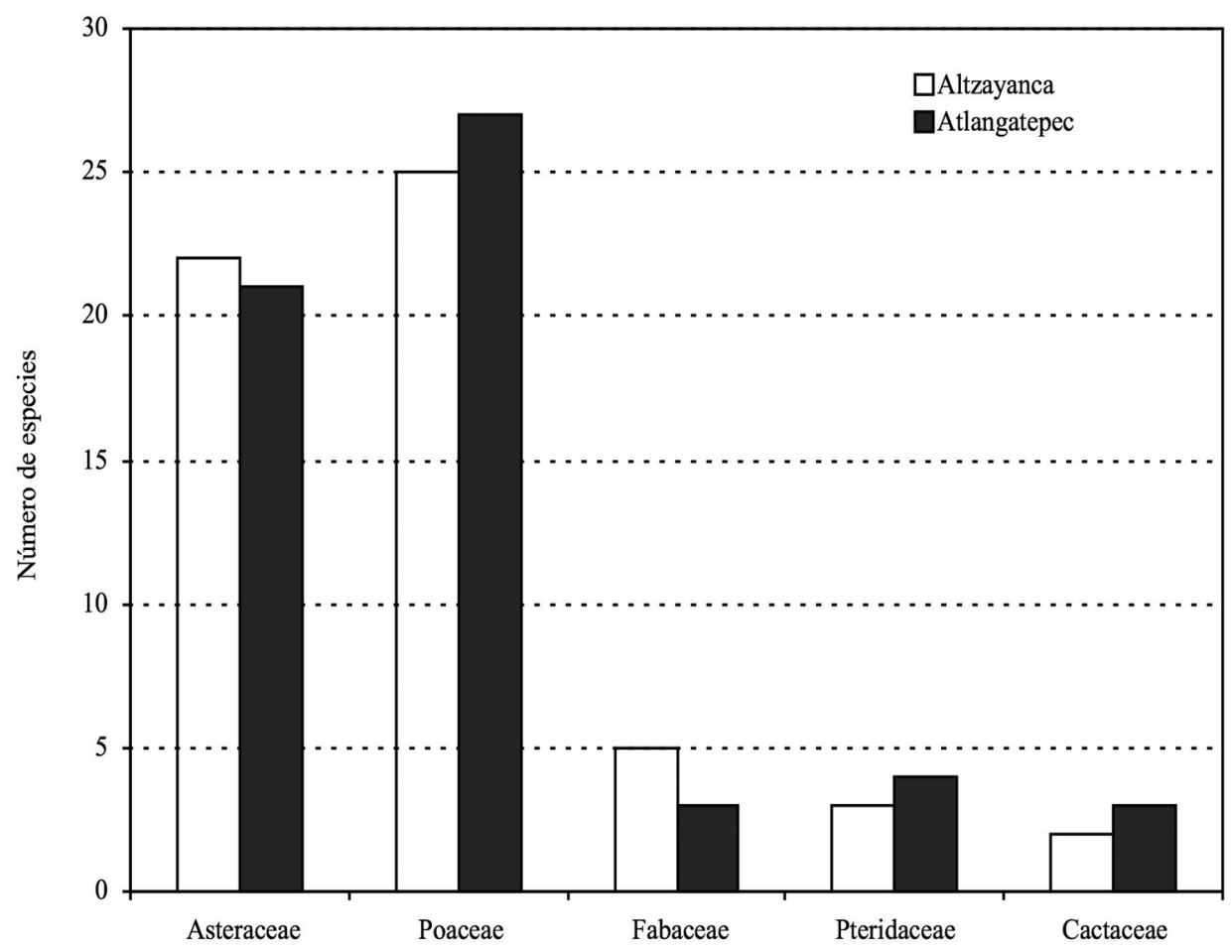

Figura 4. Número de especies de las familias representativas. 

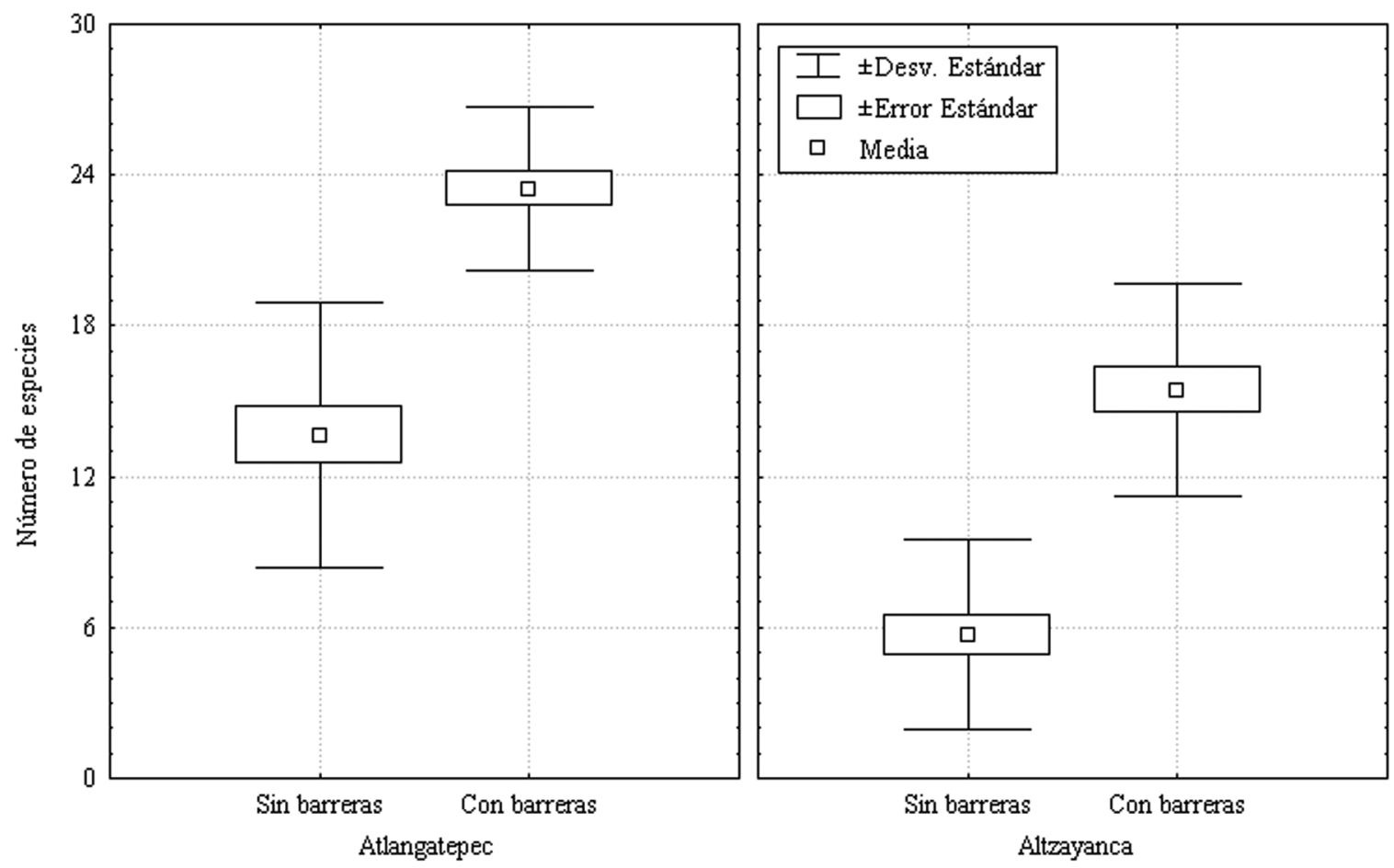

Figura 5. Diagrama de cajas y alambres del número total de especies observadas en cada unidad experimental con diferencias significativas entre localidades, y con barreras y sin ellas $(F=60.32765, \mathrm{p}<0.00001)$.

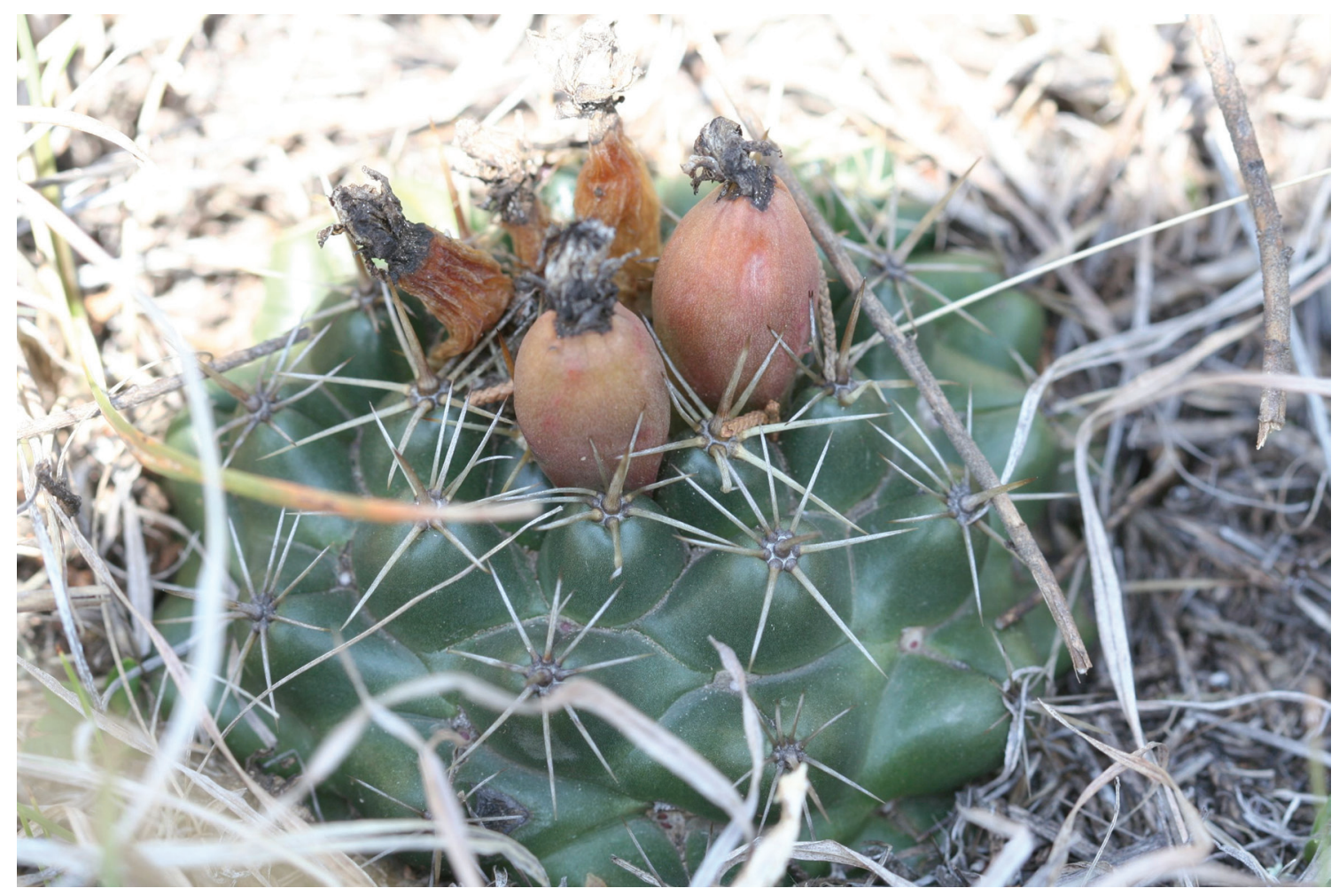

Figura 6. Vista de Coryphanta bussleri (Mundt) Scheinvar. 
(Fig. 3). Cada una de las 2 unidades experimentales de Altzayanca que se resuelven independientes (marcadas como grupo 1 y 2 en la Fig. 3) presentaron durante todo el estudio una sola especie: Piptochaetium seleri para ALR1 y Aristida mexicana para ALR13, aunque éstas no son exclusivas para dicha localidad. Ambas unidades experimentales se encuentran en sitios muy deteriorados y llama la atención que las plantas que allí se desarrollan pertenezcan al grupo de las gramíneas (Poaceae), las cuales son muy conocidas por su facilidad para asociarse con microorganismos y formar agregados en suelos tepetatosos (Acevedo-Sandoval et al., 2001; Betancourt et al., 1999, 2000; Loredo-Osti et al., 2004). Los grupos 3 y 4 relacionan a unidades experimentales de la misma localidad, no importando si son las que tienen barreras o no o si estan contiguas.

La diferencia en la presencia de especies entre las parcelas con barreras y las que no las presentan es muy marcada; por tal motivo, uno de los mecanismos que pueden llegar a facilitar la recuperación de estos suelos es la elaboración y colocación de barreras físicas que disminuyan el acarreo de suelo por la erosión hídrica y el consecuente azolve de los cuerpos de agua a donde llegan (Guerra, 2005). Además, el crecimiento de plantas en estos suelos se ha documentado que mejora su estructura por la formación de agregados, incorporan materia orgánica que eleva su fertilidad, permite mayor infiltración de agua y menor escurrimiento superficial, disminuyendo la erosión y son capaces de asociarse a microorganismos que les permiten sobrevivir en condiciones extremas (Álvarez-Solís et al., 2000; Acevedo-Sandoval et al., 2001; Velázquez-Rodríguez et al., 2001; Baéz-Pérez et al., 2002; García-Cruz et al., 2007, 2008; Haulon et al., 2007; Pajares-Moreno et al., 2010).

Por otro lado, las condiciones ambientales presentes en estos sitios degradados, favorecen el desarrollo de especies adaptadas a condiciones extremas, como Coryphanta bussleri (Fig. 6). La presencia de esta especie llama la atención ya que en este caso representa una nueva población y puede tener potencial uso ornamental debido a sus flores vistosas (Rzedowski de y Rzedowski, 2005).

\section{Agradecimientos}

Los autores agradecen A FOMIX (CONACyTGobierno del estado de Tlaxcala) el apoyo económico otorgado para la realización del presente trabajo a través del proyecto "Uso de árboles y arbustos nativos micorrizados para la restauración de suelos degradados" con clave FOMIX-TLAX-2004-20; así también, la revisión exhaustiva del manuscrito a los revisores anónimos que permitieron enriquecer la información aquí contenida.

\section{Literatura citada}

Acevedo-Sandoval, O., A. Velázquez-Rodríguez y D. FloresRomán. 2001. Agregación por especies vegetales y abonos orgánicos en tepetates fracturados en condiciones de invernadero. Terra Latinoamericana 19:363-373.

Acosta, P. R., G. L. Galindo F., L. V. Hernández C., A. EstradaTorres, A. Kong-Luz, A. Montoya-Esquivel y G. SantiagoMartínez. 1991. Listado preliminar de la flora fanerogámica y micológica del estado de Tlaxcala. Universidad Autónoma de Tlaxcala-Jardín Botánico de Tizatlán, Gobierno del Estado de Tlaxcala, Tlaxcala. $79 \mathrm{p}$.

Álvarez-Solís J. D., R. Ferrera-Cerrato y J. D. Etchevers-Barra. 2000. Actividad microbiana en tepetate con incorporación de residuos orgánicos. Agrociencia 34:523-532.

Baéz-Pérez A., J. D. Etchevers-Barra, C. Hidalgo-Moreno, Ch. Prat, V. Ordaz-Chaparro y R. Núñez-Escobar. 2002. $\mathrm{C}$ orgánico y $\mathrm{P}$ Olsen en tepetates cultivados de México. Agrociencia 36:643-653.

Baroni-Urbani, C. y M. W. Buser. 1976. Similarity of binary data. Systematic Zoology 25:251-259.

Betancourt Y. P., J. González R., B. Figueroa S. y F. González C. 1999. Materia orgánica y caracterización de suelos en proceso de recuperación con coberturas vegetativas en zonas templadas de México. Terra Latinoamericana 17:139-148.

Betancourt Y., P., J. L. Oropeza M., B. Figueroa S., V. Ordaz Ch., C. Ortiz S. y A. Hernández G. 2000. Pérdidas de suelo y potencial hidrológico en parcelas con coberturas vegetativas de especies forrajeras. Terra Latinoamericana 18:263-275.

Cain, M. L., B. G. Milligan y A. E. Strand. 2000. Long-distance seed dispersal in plant populations. American Journal of Botany 87:1217-1227.

Campos-Mota, L., G. A. Baca-Castillo, D. Jaén-Contreras, A. Muratalla-Lúa y R. Acosta-Hernández. 2004. Fertirriego y micorriza en frambuesa roja cultivada en tepetate. Agrociencia 38:75-83.

Castillejos, C. C., E. Solano C. y E. Ángeles. 2007. Florística del estado de Tlaxcala. In Biodiversidad de la Faja Volcánica Transmexicana, I. Luna, J. J. Morrone y D. Espinosa (eds.). Universidad Nacional Autónoma de México, México, D.F. p. 255-271.

Davidse G., M. Sousa S. y A.O. Chater (eds.). 1994. Flora mesoamericana. Alismataceae a Cyperaceae, vol 6 . Universidad Nacional Autónoma de México / Missouri Botanical Garden / The Natural History Museum, México, D.F. 543 p.

Gama-Castro, J., E. Solleiro-Rebolledo, D. Flores-Román, S. Sedov, H. Cabadas-Baéz y J. Díaz-Ortega. 2007. Los tepetates y su dinámica sobre la degradación y el riesgo ambiental: El caso del glacis de Buenavista, Morelos. Boletín de la Sociedad Geológica Mexicana 59:133-145.

García-Cruz, A., D. Flores-Román, N. E. García-Calderón, 
R. Ferrera-Cerrato y A. S. Velázquez-Rodríguez. 2007. Habilitación de un tepetate por efecto de mejoradores biológicos. Agrociencia 41:723-731.

García-Cruz, A., D. Flores-Román, N. E. García-Calderón y R. Ferrera-Cerrato. 2008. Efecto de enmiendas orgánicas, higuera y micorriza sobre las características de un tepetate. Terra Latinoamericana 26:309-315.

González-Chávez, M. C. A., M. C. Gutiérrez-Castorena y S. Wright. 2004. Hongos micorrízicos arbusculares en la agregación del suelo y su estabilidad. Terra Latinoamericana 22:507-514.

Guerra, V. 2005. Funcionamiento y conservación de una Cuenca Hidrográfica. Importancia y manejo del bosque. In La edafología y la sostenibilidad. XXIII Curso Diplomado Internacional de Edafología, G. Flores G., E. Fuentes R., N. García C., M. Haulon y A. Vera R. (comps.). Universidad Autónoma de Tlaxcala, Tlaxcala. p 50-62.

Haulon M., G. Werner, G. Flores-García, A. Vera-Reyes y P. Félix-Henningsen. 2007. Assesment of erosion rates during rehabilitation of hardened volcanic soils (tepetates) in Tlaxcala. Revista Mexicana de Ciencias Geológicas 24:498-509.

INEGI (Instituto Nacional de Estadística, Geografía e Informática). 2003. Información Nacional sobre perfiles de suelo, versión 1.2. México, D.F. 25 p.

Kovach W.L. 1999. MVSP-A MultiVariate Statistical Package for Windows, ver. 3.1 Kovach Computing Services, Pentraeth, Wales, Great Britain.

López, D. J., R. Acosta P. y A. I. Sánchez H. 2005. Plantas. In Biodiversidad del Parque Nacional Malinche Tlaxcala México, J. Fernández F. y J. López D. (comps.). Coordinación General de Ecología-Gobierno del Estado de Tlaxcala. p. 73-99.

Loredo-Osti., C., L. López-Reyes y D. Espinosa-Victoria. 2004. Bacterias promotoras del crecimiento vegetal asociadas con gramíneas: una revisión. Terra Latinoamericana 22:225-229.

McVaugh, R. 1992. Gymnosperns and Pteridophytes. In Flora Novo-Galiciana. A descriptive account of the vascular plants of western Mexico, vol. 17. , R. Anderson (ed.). The University of Michigan Herbarium. 467 p.

Návar, J. y T. J. Synnott. 2000. Surface runoff, soil erosion and land use in northeastern Mexico. Terra Latinoamericana 18:247-253.

Navarro-Garza, H., A. Pérez-Olvera y D. Flores-Sánchez. 2004. Productividad del tepetate con sistemas rotacionales. Terra Latinoamericana 22:71-79.

Pajares-Moreno S., J. F. Gallardo-Lancho, S. Marinari y J. D.
Etchevers-Barra. 2010. Indicadores bioquímicos de calidad de tepetates cultivados del Eje Neovolcánico Mexicano. Agrociencia 44:121-134.

Pérez, C. L. 2009. Microorganismos presentes en el proceso de rehabilitación de suelos degradados en los municipios de Altzayanca y Atlangatepec, Tlaxcala.Tesis, Facultad de Agrobiología. Universidad Autónoma de Tlaxcala, Ixtacuixtla. $99 \mathrm{p}$.

Prat, Ch., V. Ordaz Ch. y J. Rugama U. 2003. Impacto de la roturación y del manejo agronómico de un tepetate sobre su estructura. Terra Latinoamericana 21:109-115.

Rodríguez, R. F. 2009. Uso de simbiontes mutualistas en plantas nativas y cultivadas usadas para la restauración de tepetates. Tesis, Facultad de Agrobiología, Universidad Autónoma de Tlaxcala, Ixtacuixtla. 65 p.

Rzedowski, G. C.de y J. Rzedowski. 2005. Flora fanerogámica del Valle de México. Instituto de Ecología, A.C./ Comisión Nacional para el Conocimiento y Uso de la Biodiversidad. México, D.F. 1406 p.

Sánchez, M. 1993. Las cactáceas del estado de Tlaxcala. Tesis, Facultad de Agrobiología, Universidad Autónoma de Tlaxcala, Ixtacuixtla. 40 p.

Santiago-Martínez, M. G. 2010. Uso de árboles y arbustos nativos micorrizados para la restauración de suelos degradados. Anexo 1. Informe final del proyecto FOMIX-TLAX 2004CO1-20. CoNaCyT, Gobierno del Estado de Tlaxcala, CONAFOR, Universidad Autónoma de Tlaxcala. 41 p.

StatSoft, 1998. Statistica for Windows. Tulsa, Oklahoma.

Tsuyuzaki, S. y M. Goto. 2001. Persistence of seed bank under thick volcanic deposits twenty years after eruptions of Mount Usu, Hokkaido Island, Japan. American Journal of Botany 88:1813-1817.

Velázquez-Rodríguez, S., D. Flores-Román y O. A. AcevedoSandoval. 2001. Formación de agregados en tepetates por influencia de especies vegetales. Agrociencia 35:311-320.

Velázquez-Rodríguez, S., D. Flores-Román, J. D. EtcheversBarra y N. E. García-Calderón. 2008. Materia orgánica en tepetate bajo cultivo de higuera y pasto, acondicionado con estiércol y fertilizante. Agrociencia 42:11-19.

Werner, G. 2005. Rehabilitación y manejo sostenible de los tepetates. In La edafología y la sostenibilidad. XXIII Curso Diplomado Internacional de Edafología, G. Flores G., E. Fuentes R., N. García C., M. Haulon y A. Vera R. (comps.). Universidad Autónoma de Tlaxcala, Tlaxcala, Tlaxcala. p. 63-73. 
Apéndice 1. Catálogo de especies de plantas vasculares recolectadas en 2 localidades con suelos de tepetates en el estado de Tlaxcala. C.V., ciclo de vida, anual (a) o perenne (p); SB, unidad experimental sin barrera; CB, unidad experimental con barrera, x, presencia, -, ausencia. 1, especies presentes en tepetates de Altzayanca pero no registradas en las unidades experimentales; 2 , especies presentes en tepetates de Atlangatepec pero no registradas en las unidades experimentales.

Familia y nombre cientifico

C. $V$.

Stenandrium dulce (Cav.) Nees 1,2

AGAVACEAE

Agave macroculmis Tod.

ALLIACEAE (LILIACEAE)

Allium glandulosum Link et Otto

Allium stoloniferum Ownbey ex T.D. Jacobsen

Milla biflora Cav. 2

AMARANTHACEAE

Amaranthus hybridus L.

AMARYLLIDACEAE

Zephyranthes brevipes Standl. 2

ANTHERICACEAE (LILIACEAE)

Echeandia flavescens (Schult. et Schult. f.) Cruden 1,2

APIACEAE

Eryngium serratum Cav.

ASCLEPIADACEAE

Asclepias linaria Cav.

ASTERACEAE

Ageratina glabrata (Kunth) R.M. King et H. Rob. (Eupatorium glabratum Kunth)

Baccharis conferta Kunth

Bahia xylopoda Greenm.

Barkleyanthus salicifolius (Kunth) H. Rob. et Brettell

(Senecio salignus DC.)

Bidens aurea (Aiton) Sherff

Bidens odorata Cav.

Brickellia veronicifolia (Kunth) A. Gray

Chaetopappa ericoides (Torr.) G.L. Nesom

Conyza canadensis (L.) Cronquist

Cosmos bipinnatus Cav. a

Localidad

\begin{tabular}{cccc}
\multicolumn{2}{c}{ Altzayanca } & \multicolumn{2}{c}{ Atlangatepec } \\
$S B$ & $C B$ & $S B$ & $C B$
\end{tabular}

a

$\mathrm{x} x$

a

a

a

$\mathrm{p}$

a

$\mathrm{p}$

a

a

$\mathrm{p}$

a

a

a
X

X

X

X

X

$\mathrm{X}$

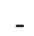

X

X

X

X

$\mathrm{X}$

X

$$
\text { - }
$$

X

X

X 
Dahlia coccinea Cav. 2

Dugesia mexicana A. Gray

Erigeron versicolor (Greenm.) G.L. Nesom

Eupatorium deltoideum Jacq.

Eupatorium scorodonioides A. Gray

Gamochaeta falcata (Lam.) Cabrera

(Gnaphalium falcatum Lam.)

Haplopappus spinulosus (Pursh) DC.

Haplopappus venetus (Kunth) S.F. Blake (Isocoma veneta (Kunth) S.F. Blake)

Pinaropappus roseus (Less.) Less.

Piqueria trinervia Cav.

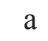

a a

$\mathrm{p}$

$\mathrm{p}$

a

$\mathrm{p}$

Pseudognaphalium semiamplexicaule (DC.) Anderb. (Gnaphalium semiamplexicaule DC.)

Sanvitalia procumbens Lam.

Senecio cinerarioides Kunth

Sonchus oleraceus L.

Stevia salicifolia Cav.

Stevia serrata Cav.

Tagetes lucida Cav.

Tagetes lunulata Ortega

Tagetes micrantha Cav.

Taraxacum officinale F.H. Wigg.

Tridax coronopifolia (Kunth) Hemsl.

$\begin{array}{lcccc}\mathrm{a} & - & \mathrm{x} & \mathrm{x} & \mathrm{x} \\ \mathrm{a} & - & - & \mathrm{x} & \mathrm{x} \\ \mathrm{p} & - & \mathrm{x} & \mathrm{x} & - \\ \mathrm{a} & \mathrm{x} & \mathrm{x} & - & \mathrm{x} \\ \mathrm{p} & - & - & - & \mathrm{x} \\ \mathrm{p} & \mathrm{x} & \mathrm{x} & \mathrm{x} & \mathrm{x} \\ \mathrm{a} & - & \mathrm{x} & - & - \\ \mathrm{a} & \mathrm{x} & \mathrm{x} & \mathrm{x} & \mathrm{x} \\ \mathrm{a} & \mathrm{x} & \mathrm{x} & \mathrm{x} & \mathrm{x} \\ \mathrm{a} & \mathrm{x} & \mathrm{x} & - & - \\ \mathrm{a} & \mathrm{x} & \mathrm{x} & \mathrm{x} & \mathrm{x}\end{array}$

\section{BRASSICACEAE}

Brassica rapa $\mathrm{L}$.

Capsella bursa-pastoris (L.) Medik.

BUDDLEJACEAE

Buddleja cordata Kunth

CACTACEAE

Coryphantha bussleri (Mundt) Scheinvar 2

Mammillaria rhodantha Link et Otto 1

Mammillaria uncinata Zucc. ex Pfeiff. 2

Opuntia streptacantha Lem. 1,2

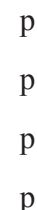

CALOCHORTACEAE (LILIACEAE)

Calochortus barbatus (Kunth) J.H. Painter 1,2 
Drymaria glandulosa Bartl.

a

$\mathrm{X}$

$\mathrm{X}$

CISTACEAE

Helianthemum glomeratum (Lag.) Lag. ex Dunal 1

Helianthemum patens Hemsl. 1

COMMELINACEAE

Tradescantia crassifolia Cav. var. acaulis (M. Martens et Galeotti) C.B. Clarke

Tripogandra purpurascens (S. Schauer) Handlos

CONVOLVULACEAE

Dichondra argentea Humb. et Bonpl. ex Willd.

Evolvulus alsinoides (L.) L.

Ipomoea capillacea (Kunth) G. Don.

CRASSULACEAE

Echeveria secunda Booth ex Lindl. 1

Villadia misera R.T. Clausen 2

CUPRESSACEAE

Juniperus deppeana Steud.

EUPHORBIACEAE

Chamaesyce hirta (L.) Millsp. 2

(Euphorbia hirta L.)

Euphorbia serpens Kunth

FABACEAE

Cologania angustifolia Kunth

Crotalaria rotundifolia J.F. Gmel.

Dalea minutifolia (Rydb.) Harms

Lessertia frutescens (L.) Goldblatt et J.C. Manning 1

(Sutherlandia frutescens (L.) R. Br.)

Macroptilium gibbosifolium (Ortega) A. Delgado

GERANIACEAE

Geranium seemannii Peyr.

\section{HYPERICACEAE}

Hypericum silenoides Juss. 1

JUNCACEAE

Juncus effusus L. 1,2

LAMIACEAE

Salvia microphylla Kunth 
Linum australe A. Heller 1

MIMOSACEAE (FABACEAE)

Mimosa biuncifera Benth.

NOLINACEAE

Nolina parviflora (Kunth) Hemsl.

ORCHIDACEAE

Schiedeella eriophora (Rob. et Greenm.) Schltr.

(Spiranthes eriophora Rob. et Greenm.)

OXALIDACEAE

Oxalis corniculata L.

Oxalis divergens Benth. ex Lindl.

PAPAVERACEAE

Argemone ochroleuca Sweet

PHYTOLACCACEAE

Phytolacca icosandra L.

PINACEAE

Pinus pseudostrobus Lindl.

PLANTAGINACEAE

Plantago linearis Kunth

Plantago nivea Kunth

POACEAE

Achnatherum editorum (E. Fourn.) Valdés-Reyna ex Barkworth (Stipa editorum E. Fourn.)

$\begin{array}{lllll}\mathrm{a} & \mathrm{x} & \mathrm{x} & \mathrm{x} & \mathrm{x} \\ \mathrm{a} & \mathrm{x} & \mathrm{x} & \mathrm{x} & \mathrm{x} \\ \mathrm{a} & - & \mathrm{x} & \mathrm{x} & \mathrm{x} \\ \mathrm{a} & - & - & \mathrm{x} & - \\ \mathrm{a} & \mathrm{x} & - & \mathrm{x} & \mathrm{x} \\ \mathrm{a} & \mathrm{x} & \mathrm{x} & \mathrm{x} & - \\ \mathrm{a} & \mathrm{x} & \mathrm{x} & \mathrm{x} & \mathrm{x} \\ \mathrm{a} & - & - & - & - \\ \mathrm{p} & \mathrm{x} & - & \mathrm{x} & \mathrm{x} \\ \mathrm{p} & - & - & - & - \\ \mathrm{p} & \mathrm{x} & \mathrm{x} & - & - \\ \mathrm{p} & - & - & - & \mathrm{x} \\ \mathrm{p} & - & - & - & - \\ \mathrm{p} & - & - & & \end{array}$

Aegopogon cenchroides Humb. et Bonpl. ex Willd.

Andropogon glomeratus (Walter) Britton, Sterns et Poggenb.

Aristida adscensionis L.

Aristida divaricata Humb. et Bonpl. ex Willd.

Aristida mexicana Scribn. ex Henrard

Aristida schiedeana Trin. et Rupr.

Bothriochloa laguroides (DC.) Herter 1,2

Bouteloua curtipendula (Michx.) Torr.

Bouteloua dactyloides (Nutt.) Columbus 2

(Buchlöe dactyloides (Nutt.) Engelm.)

Bouteloua hirsuta Lag.

Bouteloua repens (Kunth) Scribn.

Bouteloua scorpioides Lag.

Bouteloua simplex Lag. 
Bromus catharticus Vahl

Chascolytrum subaristatum (Lam.) Desv.

Cynodon dactylon (L.) Pers.

Eragrostis curvula (Schrad.) Nees

Eragrostis intermedia Hitchc.

Eragrostis mexicana (Hornem.) Link 2

Eragrostis pilosa (L.). P. Beauv. 1

Eragrostis tenuifolia (A. Rich.) Hochst. ex Steud. 1,2

$\begin{array}{lcccc}\mathrm{a} & - & \mathrm{x} & - & \mathrm{x} \\ \mathrm{a} & - & - & - & \mathrm{x} \\ \mathrm{p} & - & - & \mathrm{x} & \mathrm{x} \\ \mathrm{a} & - & \mathrm{x} & - & \mathrm{x} \\ \mathrm{a} & \mathrm{x} & \mathrm{x} & \mathrm{x} & \mathrm{x} \\ \mathrm{a} & - & - & - & - \\ \mathrm{a} & - & - & - & - \\ \mathrm{a} & - & - & - & - \\ \mathrm{p} & - & \mathrm{p} & \mathrm{x} & \mathrm{x} \\ \mathrm{a} & \mathrm{x} & \mathrm{x} & \mathrm{x} & \mathrm{x} \\ \mathrm{a} & - & \mathrm{x} & - & - \\ \mathrm{a} & \mathrm{x} & \mathrm{x} & - & - \\ \mathrm{a} & - & - & - & -\end{array}$

Muhlenbergia distans Swallen 1

Muhlenbergia dubia E. Fourn. 1

(Muhlenbergia firma Beal)

Muhlenbergia fragilis Swallen

Muhlenbergia glauca (Nees) B.D. Jacks. 1

Muhlenbergia macroura (Kunth) Hitchc. 1

Muhlenbergia minutissima (Steud.) Swallen

Muhlenbergia ramulosa (Kunth) Swallen 1

Muhlenbergia rigida (Kunth) Kunth 2

Nassella tenuissima (Trin.) Barkworth

Pennisetum clandestinum Hochst. ex Chiov.

Piptochaetium seleri (Pilg.) Henrard

Poa annua L.

Schizachyrium sanguineum (Retz.) Alston

POLEMONIACEAE

Loeselia caerulea (Cav.) G. Don.

POLYGONACEAE

Rumex acetosella L.

PTERIDACEAE

Cheilanthes bonariensis (Willd.) Proctor

Cheilanthes lendigera (Cav.) Sw.

Cheilanthes myriophylla Desv. 1,2

Pellaea ternifolia (Cav.) Link 


\section{RHAMNACEAE}

Adolphia infesta (Kunth) Meisn.

\section{RUBIACEAE}

Bouvardia ternifolia (Cav.) Schltdl.

Richardia scabra L.

Richardia tricocca (Torr. et A. Gray) Standl.

\section{SCROPHULARIACEAE}

Castilleja tenuiflora Benth. 1

Bacopa procumbens (Mill.) Greenm. 1,2

Penstemon barbatus (Cav.) Roth

Verbascum thapsus L. 2

SOLANACEAE

Physalis philadelphica Lam.

Solanum americanum Mill.

VERBENACEAE

Verbena gracilis Desf. 2 
